\title{
Recursos verbales de expresión de duda en la etapa de confrontación en discusiones orales en la sala de clases $^{*}$
}

\author{
Juan Salazar Parra**
}

\section{Resumen}

En el marco de la teoría pragma-dialéctica, la argumentación es un proceso en el cual se resuelve una diferencia de opinión. El modelo ideal para resolver dicha diferencia se denomina 'discusión crítica' e, idealmente, posee cuatro fases de desarrollo: confrontación, apertura, argumentación y clausura (van Eemeren \& Grootendorst, 1992). En este estudio nos centraremos en la etapa de confrontación y se intentará identificar los recursos verbales empleados en la etapa de confrontación, comparando, además, dichos recursos entre diversas discusiones orales. Cabe señalar que los estudiantes no dan cuenta suficientemente de la etapa de confrontación y, por ello, su discurso evidencia un escaso uso de recursos lingüístico-discursivos que den cuenta del planteamiento de la disputa o de la toma de posición, especialmente en aquellas temáticas relacionadas con la bioética.

Palabras clave: Discusión oral, discusión crítica, etapa de confrontación, expresión de duda.

\section{Abstract}

In pragma-dialectics, argumentative discourse is a process which solves a difference of opinion. The ideal model for resolving this difference is called 'critical discussion' and presents four stages: confrontation, opening, argumentation, and closing (van Eemeren \& Grootendorst, 1992). In this study we will focus on the confrontation stage and we will try to identify the resources used by students comparing different oral discussions. It should be noted that students do not account sufficiently this phase and thus their discourse evidence a low use of linguistic-discursive resources to approach the dispute, especially those topics related to bioethics.

Key words: Oral discussion, critical discussion, confrontation stage, expression of doubt.

* Inserto en el marco del proyecto FONDECYT № 1060439 "La competencia argumentativa oral en la sala de clases: Un estudio exploratorio con estudiantes de enseñanza media".

** Pontificia Universidad Católica de Valparaíso. juan.salazar.p@mail.ucv.cl 


\section{Introducción}

La argumentación está permanentemente presente en nuestras vidas: en las discusiones con amigos y familiares, en la publicidad que nos rodea, en la escuela, en los debates públicos, en los periódicos, en la televisión, en los tribunales, etc. El estudio de esta modalidad discursiva se ha centrado en la argumentación como razonamiento práctico (Perelman \& Olbrechts-Tyteca, 1958), como tipo de acto de habla (van Eemeren \& Grootendorst, 1992) y como forma lógica (Toulmin, 1958, 2001). Solo recientemente se ha abordado, específicamente, los recursos lingüísticos propios de la argumentación en tanto unidad dialógica (van Eemeren, Houtlosser \& Snoeck, 2007).

En este último sentido, la argumentación puede concebirse como un instrumento cultural que le permite a los sujetos adquirir un desempeño verbal coherente con las funciones ciudadanas (cognitivas y socioculturales) que los diversos contextos demanden, dando oportunidad para "la elección de un punto de vista personal respecto de algún tema polémico" y apreciando "el aporte de estas actitudes para la formación personal y la convivencia democrática" (MINEDUC, 2004).

Se puede indicar, entonces, que para lograr la tarea central de la argumentación, esta es la de convencer al antagonista y solucionar la disputa inicial, se emplearán diversos actos de habla y recursos verbales que acompañarán las etapas de la discusión y permitirán a los debatientes permanecer en el debate y visualizar alguna resolución o cierre del mismo (van Eemeren \& Grootendorst, 1992). Entre los variados procedimientos verbales que emplearían los sujetos para hacer que el oponente desista de su punto de vista y adhiera al contrario, se encuentra en primera jerarquía, el uso de recursos verbales que indiquen la modalidad epistémica de duda. En otras palabras, se pone en duda por medios verbales el punto de vista opuesto. 
Ahora bien, el estudio de los recursos verbales que indiquen duda no ha sido fructífero en lengua española, ni menos en el discurso argumentativo oral. Algunas investigaciones se han centrado en el estudio específico de las formas del subjuntivo (Hui-Chuan, 2000; Lu, 2003) o en una reducción de estas formas a la categoría de 'verbos de comunicación' (Bosani, 2000). Se ha obviado en estas investigaciones que estos verbos y modos verbales, en conjunto, introducen un significado conjetural más que uno asertivo a las proposiciones que lo siguen (Hyland, 1998; Sabaj, 2004) y que, por ello, son de relevancia en el estudio de la argumentación oral y de la resolución de disputas frente a puntos de vista divergentes.

En definitiva, a partir de estos antecedentes de la problemática en cuestión, se pueden plantear ciertas interrogantes a las que se intentará dar solución en este estudio: ¿qué recursos verbales se emplean en el planteamiento de un punto de vista? ¿Qué verbos y modos verbales se utilizan para conjeturar un punto de vista opuesto? ¿De qué manera influye la temática de la discusión en ciertos recursos verbales para expresar las dudas con respecto a las aseveraciones u opiniones divergentes?

Por tanto, los objetivos de esta pesquisa son identificar los recursos verbales empleados en las distintas etapas de la discusión crítica y comparar los recursos verbales utilizados en una discusión espontánea y en una dirigida.

Este escrito consta de seis apartados. En el primero se indican los conceptos claves del estudio de la argumentación desde la perspectiva de la pragma-dialéctica y de los indicadores verbales de duda asociados a la etapa de confrontación. Luego, se señalan los objetivos y el corpus a analizar en este estudio. Así, en tercer lugar, se indica la metodología de trabajo. Además, se presentan los datos y una breve discusión de ellos. Finalmente, se muestran algunos comentarios finales a esta investigación.

\section{Marco de Referencia}

En este apartado, se presenta brevemente la reflexión en torno a la argumentación como práctica dialógica desde la perspectiva de la pragma-dialéctica y una alusión a los estudios de recursos verbales que se han realizado para explicar los fenómenos de dudas en la etapa de confrontación, en particular, y en la lengua, en general. 


\subsection{El discurso argumentativo desde la pragma-dialéctica}

Entre las ya clásicas teorías contemporáneas acerca de la argumentación -la nueva retórica de Perelman y Olbrechts-Tyteca (1958), la teoría de la lógica cotidiana de Toulmin (1958) y la argumentación en la lengua de Anscombre y Ducrot (1976), entre otras- destaca la perspectiva pragmadialéctica de van Eemeren y Grootendorst (1992), puesto que ofrece un modelo ideal de discusión crítica que permite tanto evaluar como interpretar y orientar la producción del discurso argumentativo.

En cuanto al discurso argumentativo oral, este se ha caracterizado por ser un diálogo en el que un sujeto-enunciador presenta una serie de razones que avalan un punto de vista y pretende modificar la conducta de otro sujeto-enunciatario, de forma que este último acepte la propuesta inicial dada por el primer participante y desista de otra visión. Es decir, en cada una de las intervenciones que realizan los sujetos participantes de la discusión, uno de ellos tratará de poner en duda el punto de vista que sostenga su oponente, en miras a que, por medio de diversas razones y empleando variadas estrategias cognitivas, retóricas, lingüísticas y socioculturales, entre otras (Marinkovich, 2007a; 2007b; Marinkovich y Salazar, 2008; Salazar, 2008), se llegue a solucionar la disputa.

A este respecto, la teoría pragma-dialéctica se convierte en una de las herramientas más eficaces para el análisis de las disputas argumentativas, ya que se considera la argumentación como "una actividad verbal, social y racional, orientada a convencer a un crítico razonable de la aceptabilidad de un punto de vista, mediante la presentación de una constelación de proposiciones que justifican o refutan la proposición expresada en el punto de vista" (van Eemeren \& Grootendorst, 2004: 12). En este sentido, la argumentación es (a) verbal, en tanto se realiza por medio del lenguaje, (b) social, es decir, que está dirigida a otras personas y (c) racional o que se basa en consideraciones intelectuales.

Desde la perspectiva de la pragma-dialéctica, la argumentación se centra en la resolución de una diferencia de opinión por medio del discurso argumentativo o, más bien, el texto discursivo, como llamamos a esa parte del discurso argumentativo en la que se persigue concretamente la resolución de una diferencia. En definitiva, un texto discursivo es "la completa constelación de expresiones (que pueden ser escritas u orales) que han sido presentadas en defensa de un punto de vista" (van Eemeren \& Grootendorst, 1992: 13).

Para resolver realmente la disputa, los puntos de vista que están en cuestión deben convertirse en el tema de una discusión crítica orientada a lograr acuerdo acerca de la aceptabilidad o inaceptabilidad de los puntos de vista en cuestión, averiguando si pueden o no ser adecuadamente 
defendidos contra las dudas o críticas (van Eemeren \& Grootendorst, 1992).

La resolución de una disputa pasa, idealmente, por cuatro etapas, que corresponden a las cuatro fases de una discusión crítica:

(a) Etapa de confrontación: Se establece que hay una disputa. Se presenta un punto de vista (PV) y es cuestionado, es decir, si se defiende un punto de vista, quiere decir que su aceptabilidad está siendo cuestionada. La persona que argumenta supone que otros dudan, o podrían dudar, de la aceptabilidad de su punto de vista. Por tanto, el propósito de su discurso es convencerlos de que tal PV es aceptable

(b) Etapa de apertura: Se toma la decisión de intentar resolver la disputa por medio de una discusión argumentativa regulada. Una parte toma el rol de protagonista (defiende el punto de vista) y la otra el de antagonista (desafía sistemáticamente al protagonista a defender su punto de vista). Ambas partes concuerdan en los puntos de partida y en las reglas de la discusión.

(c) Etapa de argumentación: El protagonista defiende su punto de vista y el antagonista lo hace presentar más argumentación, si tiene más dudas.

(d) Etapa de clausura: Se establece si se ha resuelto la disputa porque el punto de vista ha sido retirado, o porque las dudas acerca de él han sido retiradas. Si el punto de vista ha sido retirado, la disputa se ha resuelto en favor del antagonista (el protagonista no está obligado a adoptar el PV opuesto a su PV original, puede modificarlo o adoptar un PV cero); si las dudas han sido retiradas, la disputa se ha resuelto en favor del protagonista (el antagonista debe aceptar el PV del protagonista).

El modelo de una discusión crítica cumple tanto una función heurística como una función crítica en el análisis y evaluación de los discursos o textos argumentativos. La función heurística es la de ser una guía para el análisis, en cuanto permite detectar e interpretar cada elemento, y cada aspecto, del discurso o texto que sea relevante para una evaluación crítica. La función crítica es la de servir de estándar para la evaluación, proporcionando normas para determinar si la interacción argumentativa es la más adecuada para solucionar la disputa.

\subsection{Los recursos verbales en la etapa de confrontación}

\subsubsection{La duda como indicador en la etapa de confrontación}

En la etapa de confrontación, con la presentación de un PV y la respuesta del antagonista, en el caso más simple, da cuenta de la expresión 
de una duda en el discurso argumentativo. Desde la perspectiva del análisis de la conversación, una expresión dubitativa evidencia asertivamente, una respuesta no-preferida (Pomerantz, 1984). Desde que dicha respuesta se genera, asume van Eemeren et al. (2007), se produce también la forma más simple de controversia: una disputa única no-mixta.

Siguiendo la perspectiva de la pragma-dialéctica, la duda se desarrolla con indicadores de reserva (del tipo "bueno" o "eh") como una característica propia de las acciones comunicativas. Incluso, podríamos asumir que se trata de formas sin contenido semántico relevante para el tema en discusión (Gille, 2001), pero con alto contenido pragmático, ya que tenderían a minimizar la imagen negativa del interlocutor (Marinkovich, 2007; Salazar, 2008).

Ahora bien, la duda se evidencia en una serie de expresiones propias, tales como: 'No estoy seguro de eso', 'puedo desavenir', 'tengo preguntas al respecto', 'no sé, (seguido de una expresión asertiva)', '¿es ese el caso?', 'me pregunto si eso será realmente cierto', entre muchas otras que van Eemeren y Grootendorst (1994) han listado.

Cuando estas expresiones son empleadas, la duda se presenta explícitamente en el discurso; es decir, la disputa única no-mixta no es ambigua. Sin embargo, en variadas ocasiones, no se emplean estas fórmulas lingüísticas, sino que se utilizan variados recursos lingüístico-discursivos que permiten expresar las dudas. Estos casos corresponden, por ejemplo, a las preguntas empleadas en el discurso y a las formas verbales que de él se desprenden.

Con respecto a las preguntas, estas pueden ser solicitudes de clarificación o de justificación. Ambos casos implican dudas en distintos ámbitos de la conversación. Mientras las solicitudes de clarificación requieren una explicación o reformulación del PV enunciado, las de justificación solicitan argumentos de mayor solidez para validar determinada postura.

También, van Eemeren et al. (2007) ha listado distintos indicadores de actitud proposicional y de modificación de expresiones. Los primeros suponen, desde la perspectiva gramatical, un sujeto enunciador (hablante) y un verbo (acción) que permiten dudar del PV opuesto y, por ello, indican, en tanto aserciones débiles, la actitud de las proposiciones emitidas (estos son los casos de 'yo creo que', 'yo supongo', 'tengo la impresión de que', etc.). Los indicadores de modificación de expresiones corresponden a ciertas fórmulas estandarizadas en la lengua (tales como 'desde mi perspectiva', 'es probable', 'en mi opinión', etc.). Cualquiera sea el caso, ambos indicadores señalan la debilidad del juicio emitido por una aserción y, por ello, expresarían la duda respecto de un PV.

Finalmente, los estudios verbales del modo subjuntivo también darían cuenta de la expresión de duda en el discurso argumentativo. Si bien, 
la pragma-dialéctica no adopta esta propuesta de corte gramatical, por centrarse en estudios pragmáticos (de actos de habla), se abordará el estudio del subjuntivo, suponiendo que cada verbo expresaría su acción pasión o estado empleando diversas modalidades (RAE, 1979).

Los estudios verbales se centran principalmente en los de corte gramatical. A este respecto, cabe señalar que todas las gramáticas presentan al verbo como una unidad central de estudio (Sabaj, 2004). Ya, Di Tullio (1997:93) señala que:

El verbo es el núcleo del predicado y, en última instancia, el núcleo último de la cláusula porque, además de contener los rasgos flexivos, determina el número y el tipo de complementos de la cláusula, incluyendo al sujeto.

La gramática tradicional, siguiendo la propuesta de RAE (1979), supone el empleo del modo subjuntivo exclusivamente en las oraciones subordinadas siempre que el verbos del período principal exprese una acción dudosa, posible, necesaria o deseada. Sin embargo, y RAE (1979) y Di Tullio (1997) así lo indican, esta acepción no es lo suficientemente general, ya que en dichos casos, el hablante podría optar por emplear el modo subjuntivo o el indicativo.

A este respecto, RAE (1979) adhiere a las propuestas de las gramáticas latinas, que realizan una distinción de formas del subjuntivo. Se distingue un subjuntivo común o dubitativo (potencial) y uno optativo. El primero comprendería las acciones pensadas como dudosas o posibles; mientras que el segundo, las necesarias o deseadas.

Dada la naturaleza de este estudio, nos ocuparemos del tipo 'subjuntivo potencial'. Por una parte, en oraciones independientes, este subjuntivo intensificaría la duda que el hablante quiera expresar. Por otra, en oraciones dependientes, RAE (1979: 456) asume que quien duda "se da cuenta de la irrealidad objetiva del juicio que formula". Tal vez, esta es la explicación para el uso preferido de subjuntivo en oraciones subordinadas (Hui-Chuan, 2000). Ahora bien, desde un punto de vista estructural, el verbo en subjuntivo se emplea cuando el antecedente es desconocido o dudoso. Luego, el estudio del subjuntivo independiente del tipo de pesquisa o de las finalidades de las investigaciones y apreciaciones (RAE, 1979; Di Tullio, 1997; Hui-Chuan, 2000; Lu, 2003), en todas, se asume la validez de la expresión dubitativa de dicha modalidad verbal. 


\section{EL ESTUDIO}

\section{1. Objetivo}

En este estudio, se intenta describir cómo argumentan oralmente los estudiantes de un tercer año medio de la asignatura Lengua Castellana y Comunicación de un establecimiento educacional particular-subvencionado y confesional de la ciudad de Valparaíso cuando desarrollan la Unidad La argumentación. Específicamente, se pretende identificar los recursos verbales que indique duda empleados en la etapa de confrontación y comparar los recursos verbales utilizados en cuatro discusiones ocurridas en el contexto de la sala de clases.

\section{2. Corpus}

El corpus lo constituyen las transcripciones de cuatro sesiones de Lengua Castellana y Comunicación en las que se debate acerca de la existencia de Dios, la clonación, las barras bravas y la jornada escolar completa. Cada una consta de 80 intervenciones en promedio, y participan entre cinco y diez estudiantes, ya que simulan un debate grupal.

\section{3. Metodología de análisis}

Por una parte, el análisis de las secuencias orales se realizó sobre la base de la trascripción de las mismas, en donde se marcaron las etapas de la discusión crítica siguiendo a van Eemeren y Grootendorst (1992). Una vez establecidas todas las etapas, se seleccionaron exclusivamente las Ilamadas 'de confrontación' y se establecieron los PV respectivos y el ataque (o PV negativo) al mismo. Por otra parte, se detallaron los recursos verbales empleados por los interlocutores en las distintas intervenciones.

Finalmente, los resultados de la aplicación de las distintas categorías de análisis se interpretaron cualitativamente, en el sentido de dar cuenta de diferencias entre la calidad de las intervenciones, tanto desde su aporte argumentativo como del uso de los variados recursos verbales en las diferentes actividades de argumentación.

\section{Análisis y discusión de los datos}

Para la pragma-dialéctica, la dimensión lógico-racional del análisis aparece integrada a la dimensión pragmática, esto es, a la actividad verbal propiamente tal en una situación particular.

En las etapas de confrontación de las cuatro discusiones seleccionadas, se presenta un primer análisis exploratorio de los recursos más 
evidentes y de una breve explicación de su empleo. Posteriormente, se intenta proveer de una explicación generalizada de los recursos lingüísticos empleados en la etapa de confrontación.

En la primera discusión analizada cuyo tema central es el aborto, se presentan dos PV opuestos. En primer lugar, un estudiante señala que "el aborto significa un espacio de libertad para la pareja" y, luego, otro alumno indica que "el aborto atenta contra los derechos básicos de las personas". La discusión ha comenzado con el empleo de un signo de reserva, por un lado, demostrando la actitud de recelo frente a la posición inquisidora de sus oponentes (van Eemeren et. al., 2007), previendo que estos podrían dudar de su PV, o bien, por su escaso dominio de la competencia argumentativa. En este último sentido, algunas características son propias del discurso oral, su espontaneidad y coloquialidad, por ejemplo (Calsamiglia \& Tusón, 1999).

En esta discusión se presentan diversos recursos verbales que indican duda. Desde ya, el empleo del verbo poder, como forma dubitativa ("el aborto puede significar"). Asimismo, indicadores de modificación de expresiones ("en determinados casos"), ya que, siguiendo la propuesta de la pragma-dialéctica, no asume una postura absoluta u objetiva respecto del fenómeno, sino que plantea su PV asumiendo, de antemano, que sus oponentes lo atacarán. El planteamiento de este PV posee tres indicadores dubitativos, los dos señalados previamente y el empleo del modo subjuntivo. Este último uso está relacionado con dos temáticas éticas de fuerza: la libertad y el castigo; sin embargo, en ninguno de los casos, en los cuales podría haber empleado formas declarativas, ya que no existía opinión previa, se utiliza un acto de habla asertivo, sino que, por el contrario, adopta la forma "sin que sea penalizado", para relacionarlo con el deseo y evidenciar que este no es universal.

Luego, en algunos casos, se han entremezclado los indicadores de actitud proposicional ("yo creo que") con las formas dubitativas de poder ("podríamos"). Esta última situación da cuenta de una cierta inseguridad del hablante, o bien, de una marcación más efectiva de la duda, ya que en la intervención previa A3 ha hecho uso de la palabra y sus actos de habla declarativos forzaron a A4 a intervenir con este enunciado.

Finalmente, esta etapa se cierra con una pregunta por justificación realizada por el estudiante que representa un moderador del debate y que, ciertamente, intenta que los exponentes de la discusión justifiquen sus aseveraciones y prueben sus PV. Esto, por lo tanto, da pie a la extensa etapa de argumentación de dicha discusión.

La segunda discusión giró en torno al comportamiento de las barras en el fútbol. Las dos posiciones opuestas se refieren a que "el trato policial 
es excesivo a la hinchada" y que "la responsable de los disturbios es la hinchada". En general, esta discusión carece de indicadores de duda. La temática en cuestión supone, al parecer, una experiencia más cercana a los estudiantes, por lo que sus expresiones se representan por medios verbales asertivos. Solo en dos ocasiones los estudiantes evidencian rastros lingüísticos de duda. Mientras A2 da cuenta del PV e inicia su intervención con el indicador de modificación de expresiones "a veces", el resto de la discusión gira en torno a los hechos consumados y no da posibilidad a la duda de los PV. En la penúltima intervención, se hace uso de la forma dubitativa del verbo poder "X puede causarle un tec". Ahora bien, otros estudios (González y Lima) han recogido los rastros de evidencialidad en el discurso y, en particular, en tres ocasiones los interlocutores se refieren a los grupos humanos como 'uno', situación que les permitiría desinvolucrarse del discurso oficial.

Cuando los estudiantes debatieron acerca de la clonación, presentaron dos puntos de vista: "la clonación es un avance científico" y "la clonación es un atentado contra la vida". En el planteamiento de estas posturas se incorpora un discurso referido, el de la ciencia. Ya que el estudiante no parece encontrarse seguro de la fuente de la que extrajo la información, emplea el modo subjuntivo, dudando de sus propias expresiones y de aquellas pertenecientes al discurso referido. Cabe señalar que al plantear el PV, el hablante emplea un signo de reserva ("eh, bueno") para explicar el procedimiento científico que intenta señalar. Las restantes intervenciones de esta etapa no presentan indicadores de duda, en este sentido son más bien actos de habla asertivos. Solo finalmente, la etapa de confrontación termina en la sexta intervención con una pregunta por clarificación, es decir, solicitando que el PV opuesto aclare o le quite los impedimentos que lo hacen oscuro o dudoso.

En cambio, la discusión respecto de la Jornada Escolar Completa (JEC) carece de PV opuesto, los estudiantes están todos alineados con la postura de "La JEC es perjudicial e innecesaria en el colegio". A pesar de lo anterior, que podría suponer la inexistencia de recursos verbales que expresen duda en la discusión, ya que no existe un oponente real al cual enfrentar dicha posición, en esta etapa sí se observan dos cuestionamientos: uno por justificación, pidiendo razones convincentes, y otro por clarificación. Ambos casos son planteados por el profesor $(P)$ que hace de moderador del debate. Esta realidad da cuenta de que, aunque entre los debatientes no se encuentra el PV(-), el profesor representa el oponente objetivo (van Eemeren \& Grootendorst, 1992). Nuevamente, la etapa de confrontación de esta discusión se ha cerrado con una pregunta por clarificación, en la que $P$ solicita de sus debatientes que aclaren su $P V y$, junto a este, las razones que esgrimen para justificarlo. Solo en una ocasión, de seis intervenciones en total, un estudiante emplea el modo 
subjuntivo, para señalar que su propuesta no es absoluta. Mientras señala que "el nivel de estrés es mayor", le concede a su oponente objetivo la justificación de "aunque no es crítico".

En todas las discusiones se han tratado temáticas que suponen diferencias de opinión que, en algún momento debieran ser resueltas. Así, las actividades que giraron en torno al aborto, las barras bravas, la clonación y la JEC, aunque controversiales, centraron sus esfuerzos en alcanzar el acuerdo (Caimi \& Erlich, 2005).

En cualquiera de estos casos, el hablante ha reaccionado frente a una crítica hecha a su PV, es decir, alguien ha puesto en duda, mediante la consecución de otro PV, su propia postura respecto de la temática abordada. Para van Eemeren y Grootendorst (1992) es característico de una discusión de este tipo que la diferencia de opinión sea el tema de la disputa y que los usuarios intenten resolverla mediante la argumentación. Es así que los cuatro PV señalados dan cuenta de una concepción de los sujetos respecto de las temáticas en cuestión que, a su vez, supone una cierta toma de posición en las disputas. No todas las disputas dieron cuenta explícitamente de los PV y -especialmente, en la última- aunque no se puede afirmar la existencia de una contraparte de la discusión, ya que la profesora remitió sus dos intervenciones a realizar preguntas, es válido afirmar que ambos diálogos intentaban poner en duda la postura de los estudiantes.

Ahora bien, en general, si un PV es defendido en cualquiera de las discusiones quiere decir que su aceptabilidad está siendo o es posible que sea cuestionada. Desde este punto de vista, se argumenta bajo el supuesto que otros dudan o podrían dudar de la aceptabilidad del PV. Así, la mayoría de las etapas de confrontación culminaron con una pregunta por clarificación o por justificación.

La manera cómo realizaron el cuestionamiento de los PV es variopinta. Por un lado, las discusiones bioéticas (aborto y clonación) generaron, por parte de los estudiantes, la necesidad de empleo de mayores recursos que evidenciaran duda (expresiones de duda, modo subjuntivo, preguntas por clarificación y justificación), en oposición a lo sucedido en torno a las barras bravas y la JEC que, a pesar de ser controversiales, y tal vez por la cercanía vivencial con los estudiantes, dejaron menos espacio temático para la duda. En este último caso, las dudas se generaron, fundamentalmente, para dar pie a la etapa de argumentación y no para refutar un PV.

Por otra parte, los signos de reserva que van Eemeren et al. (2007:46) define como "marks [...] which are used to keep one's options open", es 
decir, disponibles para ser empleadas en otros momentos de la discusión, no poseen esta función en las discusiones analizadas. En dos ocasiones, los estudiantes comienzan sus discursos con signos de reservas, ambas pertenecen a temáticas bioéticas y dan cuenta, por un lado, de la espontaneidad del discurso oral y, por otro, de la dificultad de acceso cognitivo a las temáticas en cuestión.

Las discusiones analizadas no llegaron a la etapa de cierre o clausura que propone van Eemeren y Grootendorst (1992) idealmente para la discusión crítica. Así, la etapa de confrontación, entendida como el planteamiento de dos PV en el que uno intenta superponerse al otro, haciéndolo dudar de su veracidad, no ha cumplido su objetivo. Los estudiantes emplean, en general, actos de habla asertivos, es decir, aseguran sus enunciados empleando aseveraciones y modos verbales indicativos. No se da pie, desde esta óptica, a la duda como un elemento fundamental, el cual, un interlocutor formado utiliza para contraponer las ideas de su oponente y darle prioridad verídica a las propias. A este respecto los mismos autores señalan que la etapa de confrontación de una disputa consiste en la expresión de las diferencias de opinión y solo cuando se ha expresado claramente la discrepancia es posible que las partes intenten resolverla a través de los caminos de la discusión crítica.

\section{Conclusiones}

En esta sección se presentan algunos comentarios finales a la búsqueda de recursos verbales que indican duda en la etapa de confrontación.

Para la pragma-dialéctica, la argumentación tiene por objetivo la resolución de diferencias de opinión. En cada discusión analizada, la diferencia planteada se intenta resolver cuando uno de los PV planteados se enfrenta con su opuesto y uno de los dos resulta veraz. Cada discusión analizada planteó un PV y su contrario. Incluso, la discusión que abordó la temática de la Jornada Escolar Completa suponía que el interlocutor real, es decir, el profesor representaba un oponente objetivo: la autoridad escolar. De este modo, las cuatro temáticas controversiales dieron cuenta de una toma de posición de los sujetos respecto de ellas.

Ahora bien, para lograr este cometido, el lenguaje dispone de una serie de recursos que el hablante puede emplear para introducir la categoría de 'duda' a la discusión. Estos indicadores marcan la función de las intervenciones o de los actos de habla en el discurso (Bolado, 2007). En este sentido, los indicadores hallados permitieron, por un lado, iden- 
tificar la función que cumplía una intervención en el marco general de la discusión y, por otro, evaluar el cierre de las discusiones.

En el primer caso, el empleo de ciertas expresiones de duda, fundamentalmente centradas en el uso de 'poder' como modalizador del discurso y de evidencialidades discursivas (como el 'uno' que engloba al sujeto en un conjunto). Asimismo, el uso de los signos de reserva no con el sentido original de poder emplear los supuestos de base a lo largo de la discusión, sino demostrando, por una parte, la coloquialidad de la discusión y, por otra, el hecho de que no se apropiaron del discurso científico referido. Luego, el funcionamiento de las preguntas por clarificación o justificación se centró, principalmente, en las últimas intervenciones de la etapa de confrontación, dando pie a la etapa de argumentación en la que, finalmente, deben darse a conocer las razones que se esgrimen para adoptar tal o cual PV. También, los estudiantes emplearon, aunque en menor grado, los indicadores de modificación de expresiones, para dar cuenta de una realidad ajena y los de actitud proposicional, para marcar su compromiso o alejamiento respecto del enunciado.

En el segundo caso, la presencia de estos recursos verbales permitió poder relacionar su uso con la etapa de cierre de las discusiones. En primer lugar, los recursos verbales que indican duda son de menor frecuencia que aquellos destinados a las aseveraciones. Esto permite determinar que, si ninguna de las cuatro sesiones analizadas pudo concluir su disputa, en gran medida se debería a que, en la etapa de confrontación, no se oponen dos PV en miras a que uno de ellos cuestione a su PV(-), sino que los hablantes se limitan a exponer dos posturas respecto de un mismo tema y, por ello, no se puede resolver la disputa inicial.

Tal como se señalara al comienzo de este estudio, esta caracterización del discurso argumentativo oral corresponde a la descripción de la competencia argumentativa que, según MINEDUC (2004), debieran manifestar los estudiantes de enseñanza media para que, no solo planteen adecuadamente sus propios puntos de vista, sino que puedan insertarse apropiadamente en la sociedad.

Dentro de las limitaciones de este estudio se halla el que solo se ha pesquisado la dimensión lingüística de una etapa de las discusiones ocurridas en contexto escolar. Los resultados variarían si se consideraran otras variables u otra propuesta teórica. Asimismo, el hecho de que solo se haya analizado una fase de la discusión crítica, permite señalar que este estudio, por tanto, resulta solo de carácter exploratorio

A modo de proyecciones, este estudio podría incorporar la relación de estos recursos verbales con recursos no-verbales y realizar una evaluación 
de la injerencia de la proxémica o la cinésica al desarrollo del discurso y, en específico, de los planteamientos dubitativos. Por otro lado, a partir de este trabajo se pueden revisar los conectores argumentativos en esta y otras etapas de la discusión oral, así como comparar el nivel de competencia que manifiestan los estudiantes a nivel secundario y nivel superior.

\section{Bibliografía}

Anscombre, J.C. \& Ducrot, O. (1976). "L'argumentation dans la langue". Langages, 42, 5-27.

Bolado, G. (2007). Educaciónético-cívica:Filosofíayciudadanía[en línea]. Disponible en: aafisevilla.blogia.com/2006/091001por-una-elaboracion-democratica-de- filosofia-yciudadania-y-educacion

Bosani, A. (2000). "Verbos de comunicación y discurso". En J. de Bustos, P. Charaudeau, J. Girón, S. Iglesias \& C. López (eds.), Lengua, discurso, texto: I simposio internacional de análisis del discurso V.I (pp.253-262). Madrid: Visor.

Caimi, G. \& Erlich, F. (2005). "Dinâmica interativa e argumentativa num debate político: Aplicação do modelo pragmadialético". Núcleo, 17 (22), 85-102.

Di Tullio, A. (1997). Manual de gramática del español. Buenos Aires: Edicial.

García, A. (1986). Manual de gramática inglesa comparada. Madrid: EDI-6.

González, C. y Lima, P. (en prensa). Estrategias de expresión de la evidencialidad en la argumentación oral en sala de clases.

Gille, J. (2001).

Pautas argumentativas en el diálogo espontáneo. Un estudio de conversaciones intra e interculturales. Tesis doctoral. Universidad de Estocolmo. Estocolmo, Suecia.

Hui-Chuan, L. (2000). Estudio de la cláusula relativa restrictiva en españoly chino. Tai-Pei: Kaun Tang International.

Hyland, K. (1998). Hedging in scientific research articles. Amsterdam: John Benjamin's Publishing Company.

Lu, H. (2003). Oraciones complejas y modo subjuntivo en español. Tapei: Kuan Tang. 
Marinkovich, J. (2007a). "Las estrategias cognitivo-retóricas y la dimensión dialéctica de la argumentación oral en una clase de lengua castellana y comunicación". Signos, 40(63), 127-146.

(2007b).

"La interacción argumentativa en el aula: fases de la argumentación y estrategias de cortesía verbal". En C. Santibáñez \& B. Riffo (Eds.), Estudios contemporáneos deArgumentación y Retórica. Concepción: Ediciones Universidad de Concepción.

y Salazar, J. (2008). "La pragma-dialéctica como una herramienta analítica de la argumentación oral en el aula". En Actas de la Conferencia Internacional de Lógica, Argumentación y Pensamiento Crítico. Santiago de Chile: UDP.

MINEDUC (2004). Lengua Castellana y Comunicación / Lenguaje y Comunicación. Programa de Estudio, Tercer Año Medio, Formación General. Educación Media. Santiago de Chile: Ministerio de Educación de Chile.

Perelman, Ch. \& Olbrechts-Tyteca, L. (1958). Traité de l'argumentation. La nouvelle rhétorique. París: PUF.

Pomerantz, A. (1984). "Agreeing and disagreeing with assessments: Some features of preferred/dispreferred turn shapes". En J. Atkinson \& J. Heritage (Eds.), Structures of social action. Studies in conversation analysis, (pp. 57-102). Cambridge: Cambridge University Press.

RAE (1979).

Sabaj, O. (2004).

Salazar, J. (2008).

Toulmin, S. (1958). (2001).

Tusón, A. (1995).
Esbozo de una nueva gramática de la lengua española. Madrid: Espasa Calpe S. A.

El comportamiento de los verbos abstractos en el corpus PUCV-2003. Tesis doctoral, Pontificia Universidad Católica de Valparaíso, Valparaíso, Chile.

"Estrategias de cortesía verbal en interacciones argumentativas ocurridas en contexto escolar". Revista Alpha, 27, 77-92.

The uses of argument. Cambridge: Cambridge University Press.

Return to reason. Cambridge, MA: Harvard University Press.

"L'aula com a escenari comunicatiu". Articles de didàctica de la llengua i la literatura, 6, 47-58. 
Recursos verbales de expresión de duda en la etapa de confrontación en discusiones orales en al sala de clases / Juan Salazar

van Eemeren, F. \& Grootendorst, R. (1992). Argumentation, communication and fallacie. A pragmadialectical perspective. New Jersey: Lawrence Erlbaum.

(1996). Waar slat dat nou weer op? De taal van het meningsverschil. Amsterdam. Contact.

(2004). A Systematic Theory of Argumentation. The pragmadialectical approach. Cambridge: Cambridge University Press.

van Eemeren, F., Houtlosser, P. \& Snoeck, A. (2007). Argumentative indicators in discourse. Amsterdam: Springer. 